\title{
Generalized Bhattacharyya and Chernoff upper bounds on Bayes error using quasi-arithmetic means
}

\author{
Frank Nielsen*†
}

\begin{abstract}
Bayesian classification labels observations based on given prior information, namely class- $a$ priori and class-conditional probabilities. Bayes' risk is the minimum expected classification cost that is achieved by the Bayes' test, the optimal decision rule. When no cost incurs for correct classification and unit cost is charged for misclassification, Bayes' test reduces to the maximum a posteriori decision rule, and Bayes risk simplifies to Bayes' error, the probability of error. Since calculating this probability of error is often intractable, several techniques have been devised to bound it with closed-form formula, introducing thereby measures of similarity and divergence between distributions like the Bhattacharyya coefficient and its associated Bhattacharyya distance. The Bhattacharyya upper bound can further be tightened using the Chernoff information that relies on the notion of best error exponent. In this paper, we first express Bayes' risk using the total variation distance on scaled distributions. We then elucidate and extend the Bhattacharyya and the Chernoff upper bound mechanisms using generalized weighted means. We provide as a byproduct novel notions of statistical divergences and affinity coefficients. We illustrate our technique by deriving new upper bounds for the univariate Cauchy and the multivariate $t$-distributions, and show experimentally that those bounds are not too distant to the computationally intractable Bayes' error.
\end{abstract}

Key words: Affinity coefficient; divergence; Chernoff information; Bhattacharrya distance; total variation distance; quasi-arithmetic means; Cauchy distributions; multivariate $t$-distributions.

\section{Introduction: Hypothesis testing, divergences and affinities}

\subsection{Hypothesis testing}

Consider the following fundamental binary hypothesis testing problem 1 : Let $X_{1}, \ldots, X_{n}$ be $n$ identically and independently distributed (IID) random variables following distribution $Q$ with support $\mathcal{X}$. We consider two (simple) hypotheses:

$$
\begin{aligned}
& H_{1} \quad: \quad Q \sim P_{1}(\text { null hypothesis }) \\
& H_{2} \quad: \quad Q \sim P_{2}(\text { alternative hypothesis })
\end{aligned}
$$

and we design a test $g\left(X_{1}, \ldots, X_{n}\right): \mathcal{X}^{n} \rightarrow\{1,2\}$ to decide which hypothesis to select. The decision region $R_{1} \subseteq \mathcal{X}^{n}$ corresponds to the set of sequences $X^{n}=\left(X_{1}, \ldots, X_{n}\right)$ mapped to $H_{1}$, and the decision region $R_{2}=R_{1}^{c}$ is the complementary region.

\footnotetext{
*Sony Computer Science Laboratories, Inc. 3-14-13 Higashi Gotanda, Shinagawa-Ku, Tokyo 141-0022, Japan. Frank.Nielsen@acm.org http://www.sonycsl.co.jp/person/nielsen/. Joseph-Louis Lagrange laboratory, Univ. Nice SophiaAntipolis, CNRS, OCA, France.

$\dagger$ Accepted manuscript to appear in Pattern Recognition Letters $(10.1016 / j . p a t r e c .2014 .01 .002)$. http://www.journals.elsevier.com/pattern-recognition-letters/ Seehttp://www.journals.elsevier.com/theoretical-computer-science

${ }^{1}$ We refer the reader to the textbooks [5, 6] for an information-theoretic background based on the method of types, and to the textbook 7 for the Bayesian setting often met in pattern recognition.
} 
To illustrate this setting, consider for example the task of distinguishing a texture 21, modeled by edglet:2 $X^{n}$ centered at 2D image lattice positions, from two textures $T_{1}$ and $T_{2}$, given by their respective edgelet probability distributions $P_{1}$ and $P_{2}$ (assuming the IID hypothesis). In practice, we observe a texture sample, that is a data set $x^{n}=\left(x_{1}, \ldots, x_{n}\right)$ sampled from $X^{n}$.

There are two kinds of error [5] associated with any test:

- Type I error (misclassification when the true hypothesis is $\left.H_{1}\right): \epsilon_{1}(n)=\operatorname{Pr}\left(g\left(X_{1}, \ldots, X_{n}\right)=2 \mid H_{1}\right)$, and

- Type II error (misclassification when the true hypothesis is $\left.H_{2}\right): \epsilon_{2}(n)=\operatorname{Pr}\left(g\left(X_{1}, \ldots, X_{n}\right)=1 \mid H_{2}\right)$

In target/noise detection theory [5], a test is called a detector, and those type I and type II errors are respectively called probability of false alarm and probability of miss.

There are two main approaches for hypothesis testing that have been developed in the literature [5, 7]: The first Neyman-Pearson approach seeks to minimize the probability of miss given the probability of false alarm, without any prior information for the hypothesis. The second Bayesian approach makes use of prior information on class- $a$ priori and class-conditional probabilities for the hypothesis. We concisely review the links between hypothesis testing and statistical distances between distributions for the first nonBayesian approach in Section 1.2, and the links between hypothesis testing and statistical similarities between distributions for the second Bayesian approach in Section 1.3.

\subsection{Statistical divergences in hypothesis testing}

The first approach asks to minimize the probability of miss $\epsilon_{2}$ (false negative) given the probability of false alarm $\epsilon_{1}$ (false positive):

$$
\min _{\epsilon_{2}} \epsilon_{1} \leq \varepsilon
$$

where $\varepsilon$ is a prescribed error threshold. In the literature, the significance level (or size) of a test is $\epsilon_{1}$ and the power of a test is $1-\epsilon_{2}$. Thus we seek to maximize the power of a test given a prescribed significance level. A key result is the Neyman-Pearson lemma [5] which states the optimality of the Likelihood Ratio Test (LRT) (or equivalently its log-likelihood ratio):

$$
\Lambda\left(X_{1}, \ldots, X_{n}\right)=\log \frac{P_{1}\left(X_{1}, \ldots, X_{n}\right)}{P_{2}\left(X_{1}, \ldots, X_{n}\right)}=\sum_{i=1}^{n} \log \frac{P_{1}\left(X_{i}\right)}{P_{2}\left(X_{i}\right)} \leq \lambda,
$$

to reject $H_{1}$ in favor of $H_{2}$ (with $\operatorname{Pr}\left(\Lambda\left(X_{1}, \ldots, X_{n}\right) \leq \lambda \mid H_{1}\right)=\epsilon_{1}$ and $\lambda=\lambda(\varepsilon)$ ). Note that the larger the $\log$-likelihood ratio, the more probable the sequence $X^{n}$ comes from $P_{1}$ (and the more likely hypothesis $H_{1}$ ). From the IDD assumption, we better analyze sequences via the method of types [21, 6]: The type $h\left(x^{n}\right)$ is the empirical probability distribution of elements of $\mathcal{X}$ (say, a discrete alphabet with $d$ letters) met in $x^{n}$. That is, for alphabet $\mathcal{X}=\left\{E_{1}, \ldots, E_{d}\right\}$, the type $h\left(x^{n}\right)$ of a sample sequence is the frequency empirical histogram of elements: $h\left(x^{n}\right)=\left(h_{1}\left(x^{n}\right), \ldots, h_{d}\left(x^{n}\right)\right)$, where $h_{i}\left(x^{n}\right)=\frac{1}{n} \sum_{j=1}^{n} \delta_{x_{j}, E_{i}}=\frac{\#\left\{x_{j}=E_{i} \mid j \in\{1, \ldots, n\}\right\}}{n}$. Observe that although there are $d^{n}$ distinct sequences of length $n$ (that is, exponential in $n$ ), there is only a polynomial number of types (bounded by $(n+1)^{d}$ since each of the $d$ elements $E_{i}$ of $\mathcal{X}$ has counting number $n_{i}=n h_{i}\left(X^{n}\right)$, an integer between 0 and $\left.n\right)$.

The log-likelihood ratio of Eq. 4 rewrites as:

$$
\Lambda\left(x_{1}, \ldots, x_{n}\right)=\sum_{i=1}^{n} \log \frac{P_{1}\left(x_{i}\right)}{P_{2}\left(x_{i}\right)}=\sum_{j=1}^{d} n h_{j}\left(x^{n}\right) \log \frac{P_{1}\left(E_{j}\right)}{P_{2}\left(E_{j}\right)} .
$$

Observe that the log-likelihood ratio can be conveniently written as an inner product between two histograms $h\left(x^{n}\right)$ and $A_{12}=\left(\log \frac{P_{1}\left(E_{1}\right)}{P_{2}\left(E_{1}\right)}, \ldots, \log \frac{P_{1}\left(E_{d}\right)}{P_{2}\left(E_{d}\right)}\right): \Lambda\left(x_{1}, \ldots, x_{n}\right)=n\left\langle h\left(x^{n}\right) \mid A_{12}\right\rangle$. In [21], the inner product $\left\langle h\left(x^{n}\right) \mid A_{12}\right\rangle$ is called the reward of sequence sample $x^{n}$.

\footnotetext{
${ }^{2}$ An edgelet is a small line segment with slope quantized to take $d$ possible directions.
} 
The expected average log-likelihood ratios with respect to $P_{1}$ and $P_{2}$ are:

$$
\begin{aligned}
& \frac{1}{n} E_{P_{1}}\left[\Lambda\left(X_{1}, \ldots, X_{n}\right)\right]=\sum_{j=1}^{d} P_{1}\left(E_{j}\right) \log \frac{P_{1}\left(E_{j}\right)}{P_{2}\left(E_{j}\right)}=\mathrm{KL}\left(P_{1}: P_{2}\right) \\
& \frac{1}{n} E_{P_{2}}\left[\Lambda\left(X_{1}, \ldots, X_{n}\right)\right]=\sum_{j=1}^{d} P_{2}\left(E_{j}\right) \log \frac{P_{1}\left(E_{j}\right)}{P_{2}\left(E_{j}\right)}=-\mathrm{KL}\left(P_{2}: P_{1}\right),
\end{aligned}
$$

where $\mathrm{KL}\left(P_{1}: P_{2}\right)=\sum_{i=1}^{d} P_{1}\left(E_{i}\right) \log \frac{P_{1}\left(E_{i}\right)}{P_{2}\left(E_{i}\right)}$ denotes the Kullback-Leibler divergence between distributions $P_{1}$ and $P_{2}$. The difference between the two expected average log-likelihood ratio is the Jeffreys divergence $J\left(P_{1}, P_{2}\right)=\mathrm{KL}\left(P_{1}: P_{2}\right)+\mathrm{KL}\left(P_{2}: P_{1}\right)$, that symmetrizes the Kullback-Leibler divergence. It follows that those Kullback-Leibler and Jeffreys measures can be interpreted as measures of separability, that is distances between distributions. Note that the KL distance is asymmetric: $\operatorname{KL}\left(P_{1}: P_{2}\right) \neq \mathrm{KL}\left(P_{2}: P_{1}\right)$. The $\mathrm{KL}$ and $\mathrm{J}$ distances are not metric because they violate the triangular inequality [5].

The probability that a sequence sample $x_{1}^{n}$ from $P_{1}$ has lower reward than a sequence sample $x_{2}^{n}$ from $P_{2}$ is bounded by (see [21], Theorem 2):

$$
(n+1)^{-d^{2}} 2^{-n B\left(P_{1}, P_{2}\right)} \leq \operatorname{Pr}\left(\left\langle h\left(x_{1}^{n}\right) \mid A_{12}\right\rangle \leq\left\langle h\left(x_{2}^{n}\right) \mid A_{12}\right\rangle\right) \leq(n+1)^{d^{2}} 2^{-n B\left(P_{1}, P_{2}\right)},
$$

where $B\left(P_{1}, P_{2}\right)$ denotes the Bhattacharrya divergence:

$$
B\left(P_{1}, P_{2}\right)=-\log \sum_{j=1}^{d} \sqrt{P_{1}\left(E_{j}\right)} \sqrt{P_{2}\left(E_{j}\right)} .
$$

Although the Neyman-Pearson lemma [5] characterizes the optimal decision test, it does not specify the threshold $\lambda$. The false alarm error $\epsilon_{1}$ and miss error $\epsilon_{2}$ probabilities decay exponentially as the sample size $n$ increases (see [21], Theorem 1). Thus in the asymptotic regime, we are rather interested in characterizing the error exponents defined as the rate of that exponential decay:

$$
\alpha=\lim _{n \rightarrow \infty}-\frac{1}{n} \log _{2} \epsilon_{1}(n), \quad \beta=\lim _{n \rightarrow \infty}-\frac{1}{n} \log _{2} \epsilon_{2}(n),
$$

where $\epsilon_{1}(n)=\operatorname{Pr}\left(g\left(X^{n}\right)=2 \mid H_{1}\right)$ and $\epsilon_{2}(n)=\operatorname{Pr}\left(g\left(X^{n}\right)=1 \mid H_{2}\right)$ (and $\epsilon_{1}(n) \approx 2^{-n \alpha}$ and $\epsilon_{2}(n) \approx 2^{-n \beta}$ ). It turns out that when minimizing the asymptotic rate of misclassification error $P_{e}=\epsilon_{1}+\epsilon_{2}$, called the probability of error, the optimal threshold is $\lambda=0$, and the error rate is the Chernoff information [5, 15]:

$$
C\left(P_{1}, P_{2}\right)=\min _{\alpha \in[0,1]} B_{\alpha}\left(P_{1}: P_{2}\right),
$$

where $B_{\alpha}$ denotes the skewed Bhattacharrya divergence:

$$
B_{\alpha}\left(P_{1}: P_{2}\right)=-\log \sum_{j=1}^{d} P_{1}\left(E_{j}\right)^{\alpha} P_{2}\left(E_{j}\right)^{1-\alpha},
$$

generalizing the Bhattacharrya divergence: $B\left(P_{1}, P_{2}\right)=B_{\frac{1}{2}}\left(P_{1}: P_{2}\right)$.

Those notions of statistical divergences can be extended to continuous distributions by replacing the discrete sum by an integral (and interpreted $\mathcal{X}$ as a continous alphabet).

We now consider the Bayesian paradigm in hypothesis testing, and show how to bound the probability of misclassification error using statistical similarity measures. 


\subsection{Statistical similarities in hypothesis testing}

The Bayesian framework of hypothesis testing assumes that we are given prior beliefs over the probabilities of the two hypothesis, and we seek to minimize the expected probability of error (also called error probability): $P_{e}=\epsilon_{1} \operatorname{Pr}\left(H_{1}\right)+\epsilon_{2} \operatorname{Pr}\left(H_{2}\right)$. In this setting, both the class a priori $\left(w_{i}>0\right)$ and the class conditional probabilities $\left(p_{i}\right)$ are known beforehand (or estimated from a training labeled sample [7]). Let $q_{1}$ and $q_{2}$ be the a posteriori probabilities derived from Bayes theorem:

$$
q_{i}(x)=\frac{w_{i} p_{i}(x)}{p(x)}
$$

where $p(x)$ is the mixture density $p(x)=w_{1} p_{1}(x)+w_{2} p_{2}(x)$ (and $w_{1}+w_{2}=1$ ). Let $C=\left[c_{i j}\right]$ be the $2 \times 2$ design matrix, with $c_{i j}$ denoting the cost of deciding $x \in C_{i}$ when $x \in C_{j}$, with $1 \leq i, j \leq 2$. Furthermore, denote by

$$
r_{1}(x)=c_{11} q_{1}(x)+c_{12} q_{2}(x),
$$

and

$$
r_{2}(x)=c_{21} q_{1}(x)+c_{22} q_{2}(x),
$$

the respective conditional costs of deciding $x \in C_{i}$, for $i \in\{1,2\}$. To classify $x$, consider the decision rule:

$$
r_{1}(x) \underset{C_{2}}{\stackrel{C_{1}}{\lessgtr}} r_{2}(x) .
$$

The conditional cost of this decision rule is:

$$
r(x)=\min \left(r_{1}(x), r_{2}(x)\right) .
$$

Bayes error $B_{e}$ (see [7], p. 57) is defined as the expected cost of this decision rule:

$$
\begin{aligned}
B_{e} & =E_{p}[r(x)], \\
& =\int p(x) \min \left(r_{1}(x), r_{2}(x)\right) \mathrm{d} x, \\
& =\int_{R_{1}}\left(c_{11} w_{1} p_{1}(x)+c_{12} w_{2} p_{2}(x)\right) \mathrm{d} x+\int_{R_{2}}\left(c_{21} w_{1} p_{1}(x)+c_{22} w_{2} p_{2}(x)\right) \mathrm{d} x,
\end{aligned}
$$

where $R_{1}=\left\{x \mid r_{1}(x) \leq r_{2}(x)\right\}$ and $R_{2}=\left\{x \mid r_{2}(x) \leq r_{1}(x)\right\}$ are the decision regions induced by the decision rule of Eq. 16. Thus Bayes test for minimum cost writes as:

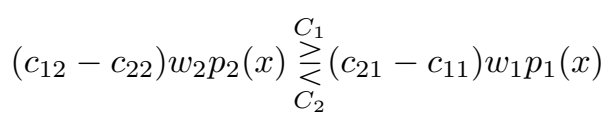

or equivalently:

$$
\frac{p_{1}(x)}{p_{2}(x)} \underset{C_{2}}{\stackrel{C_{1}}{\lessgtr}} \frac{w_{2}\left(c_{12}-c_{22}\right)}{w_{1}\left(c_{21}-c_{11}\right)} .
$$

The term $l(x)=\frac{p_{1}(x)}{p_{2}(x)}$ is called the likelihood ratio. It is equivalent and often mathematically simpler to consider the test using the log-likelihood ratio (e.g., think of the multivariate Gaussian class-conditional probabilities):

$$
\log p_{1}(x)-\log p_{2}(x) \underset{C_{2}}{\stackrel{C_{1}}{\lessgtr}} \log \frac{w_{2}\left(c_{12}-c_{22}\right)}{w_{1}\left(c_{21}-c_{11}\right)}
$$


The function $h(x)=\log p_{1}(x)-\log p_{2}(x)-\frac{w_{2}\left(c_{12}-c_{22}\right)}{w_{1}\left(c_{21}-c_{11}\right)}$ is called the discriminant function.

For symmetrical cost function $c_{21}-c_{11}=c_{12}-c_{22}$, the expected cost is called the probability of error $P_{e}$. For the probability of error, we do not incur a cost for correctly classifying and pay a unit cost for misclassification. The design matrix for the probability of error is therefore:

$$
C=\left[\begin{array}{ll}
0 & 1 \\
1 & 0
\end{array}\right]
$$

As stated earlier, the probability of error can be decomposed as the sum of two misclassification costs:

with

$$
P_{e}=w_{1} \epsilon_{1}+w_{2} \epsilon_{2}
$$

$$
\epsilon_{1}=\int_{R_{2}} p_{1}(x) \mathrm{d} x, \quad \epsilon_{2}=\int_{R_{1}} p_{2}(x) \mathrm{d} x
$$

with $R_{1}=\left\{w_{2} p_{2}(x) \leq w_{1} p_{1}(x)\right\}$ and $R_{2}=\left\{x \mid w_{1} p_{1}(x) \leq w_{2} p_{2}(x)\right\}$.

In practice, Bayes error and the probability of error are quite tricky to calculate as we need to compute integrals on decision regions $R_{1}$ and $R_{2}$. Even if those domains can be expressed simply, say for Gaussians, it is often intractable to compute analytically those integrals (e.g., for multivariate class-conditional probabilities). See the Appendix for a review of formula when class-conditional distributions are Gaussians. Therefore, we need to set good lower and upper bounds to characterize Bayes error $B_{e}$ (or the probability of error $P_{e}$ ).

A first upper bound on the probability of error $P_{e}$ is the Bhattacharrya bound [10]:

$$
P_{e} \leq \sqrt{w_{1} w_{2}} \times \rho\left(p_{1}, p_{2}\right),
$$

with $\rho\left(p_{1}, p_{2}\right)=\int \sqrt{p_{1}(x) p_{2}(x)} \mathrm{d} x$ denoting the Bhattacharrya coefficient. This first upper bound was tightened by Chernoff [4] as follows: We have

$$
P_{e} \leq w_{1}^{\alpha} w_{2}^{1-\alpha} \times \rho_{\alpha}\left(p_{1}, p_{2}\right),
$$

with $\rho_{\alpha}\left(p_{1}, p_{2}\right)=\int\left(p_{1}(x)\right)^{\alpha}\left(p_{2}(x)\right)^{1-\alpha} \mathrm{d} x$, the $\alpha$-Chernoff coefficient defined for $\alpha \in(0,1)$. Therefore the tightest upper bound is:

$$
P_{e} \leq w_{1}^{\alpha^{*}} w_{2}^{1-\alpha^{*}} \times \rho_{*}\left(p_{1}, p_{2}\right),
$$

with $\rho_{*}$ the Chernoff coefficient obtained from the following minimization problem:

$$
\rho_{*}\left(p_{1}, p_{2}\right)=\min _{\alpha \in[0,1]} \rho_{\alpha}\left(p_{1}, p_{2}\right),
$$

where $\alpha^{*} \in[0,1]$ denotes the optimal value that minimizes $\rho_{\alpha}\left(p_{1}, p_{2}\right)$. Note that the Bhattacharyya coefficient is a particular case of the Chernoff $\alpha$-coefficient (obtained for $\alpha=\frac{1}{2}$ ): $\rho=\rho_{\frac{1}{2}}$.

The Bhattacharyya, $\alpha$-Chernoff and Chernoff coefficients $\rho, \rho_{\alpha}$ and $\rho_{*}=\rho_{\alpha^{*}}$ can be interpreted a similarity measures between distributions defined by measuring the degree of overlap of their densities. Those coefficients are also called affinities.

Remark 1 (Affinity coefficients and divergences) The Chernoff $\alpha$-coefficient $\rho_{\alpha}$, the Chernoff coefficient $\rho_{*}$ and the Bhattacharrya coefficient $\rho\left(\rho=\rho_{\frac{1}{2}}\right)$ provide upper bounds on $P_{e}: 0<P_{e} \leq \frac{1}{2} \rho_{*} \leq \frac{1}{2} \rho \leq \frac{1}{2}$ (for $w_{1}=w_{2}=\frac{1}{2}$, see Eq. 29, and Eq. 27). We can transform any affinity coefficient $0<A \leq 1$ into a corresponding divergence by applying a monotonously increasing function $f$ to $\frac{1}{A}$ (with $\frac{1}{A} \in[1, \infty)$ ). By choosing $f(x)=\log (x)$, we end-up with the traditional divergences between statistical distributions: $\alpha$-Chernoff divergence, Chernoff divergence (also called Chernoff information), and Bhattacharrya divergence.

Recall that those upper bounds are useful if they can be computed easily from closed-form formula (which is not the case of $B_{e}$ nor $P_{e}$ ). 


\subsection{Closed-form Bhattacharrya/Chernoff coefficients for exponential families}

Many usual distributions like Gaussians, Poisson, Dirichlet or Gamma/Beta, etc. distributions are exponential families in disguise [11, 16] for which the skewed affinity coefficient $\rho_{\alpha}$ (i.e., similarity distance within $[0,1])$ can be computed in closed-form. An exponential family is a family $\mathcal{F}$ of distributions:

$$
\mathcal{F}=\left\{p(x ; \theta)=\exp \left(x^{\top} \theta-F(\theta)\right) \mid \theta \in \Theta\right\},
$$

indexed by a parameter $\theta \in \Theta$. Space $\Theta$ is the parameter domain, called the natural parameter space [16]. $F$ is a strictly convex and differentiable convex function called the log-normalizer. For example, the multivariate normal (MVN) distributions of mean $\mu$ and covariance matrix $\Sigma$ are exponential families for parameter $\theta=\left(\theta_{1}=\Sigma^{-1} \mu, \theta_{2}=\frac{1}{2} \Sigma^{-1}\right) \in \mathbb{R}^{d} \times S_{d}+$ (where $S_{d}^{+}$denotes the space of symmetric positive definite $d \times d$ matrices). The function $F$ (called log-normalizer) expressed in the natural coordinate system is [15:

$$
F_{\mathrm{MVN}}\left(\theta_{1}, \theta_{2}\right)=\frac{1}{2} \theta_{1}^{\top} \theta_{2}^{-1} \theta_{1}-\log \left|\theta_{2}\right|
$$

where $|\cdot|$ denotes the determinant for a matrix operand.

Wlog., let the class-conditional probabilities $p_{1}$ and $p_{2}$ belong to the same exponential family, then we have [11, 16]:

$$
\rho_{\alpha}\left(p_{1}, p_{2}\right)=e^{-J_{F}^{(\alpha)}\left(\theta_{1}, \theta_{2}\right)},
$$

where $J_{F}^{(\alpha)}\left(\theta_{1}, \theta_{2}\right)$ denote the Jensen skewed divergence [16]:

$$
J_{F}^{(\alpha)}\left(\theta_{1}, \theta_{2}\right)=\alpha F\left(\theta_{1}\right)+(1-\alpha) F\left(\theta_{2}\right)-F\left(\alpha \theta_{1}+(1-\alpha) \theta_{2}\right) \geq 0 .
$$

For example, let us consider the multivariate Gaussian family. Then, we get the Chernoff $\alpha$-coefficient:

$$
\rho_{\alpha}^{\mathrm{MVN}}\left(p_{1}, p_{2}\right)=\frac{\left|\Sigma_{1}\right|^{\frac{\alpha}{2}}\left|\Sigma_{2}\right|^{\frac{1-\alpha}{2}}}{\left|\alpha \Sigma_{1}+(1-\alpha) \Sigma_{2}\right|^{\frac{1}{2}}} \exp \left(-\frac{\alpha(1-\alpha)}{2} \Delta \mu^{\top}\left(\alpha \Sigma_{1}+(1-\alpha) \Sigma_{2}\right) \Delta \mu\right),
$$

with $\Delta \mu=\mu_{2}-\mu_{1}$. Therefore the Chernoff $\alpha$-divergence, $D_{\alpha}^{\mathrm{MVN}}\left(p_{1}, p_{2}\right)=-\log \rho_{\alpha}^{\mathrm{MVN}}\left(p_{1}, p_{2}\right)$ :

$$
D_{\alpha}^{\mathrm{MVN}}\left(p_{1}, p_{2}\right)=\frac{1}{2} \log \frac{\left|\alpha \Sigma_{1}+(1-\alpha) \Sigma_{2}\right|}{\left|\Sigma_{1}\right|^{\alpha}\left|\Sigma_{2}\right|^{1-\alpha}}+\frac{\alpha(1-\alpha)}{2} \Delta \mu^{\top}\left(\alpha \Sigma_{1}+(1-\alpha) \Sigma_{2}\right) \Delta \mu
$$

Setting $\alpha=\frac{1}{2}$, we get the Bhattacharrya divergence.

In general, we do not have a closed-form solution for finding the optimal $\alpha^{*}$ yielding the Chernoff coefficient/information. Nevertheless, the optimal value $\alpha^{*}$ of $\alpha$ can be exactly characterized [15] using the differential-geometric structure of the statistical manifold of the class-conditional distributions, and yields a fast algorithm to arbitrarily finely approximate Chernoff information $\rho_{*}$ for members of the same exponential family.

\subsection{Outline}

This paper is organized as follows: In Section 2 we show how Bayes error is related to the total variation distance. Section 3 presents our generalization of Bhattacharrya and Chernoff upper bounds relying on generalized weighted means. Section 4 illustrates several applications of the technique yielding novel upper bounds for various distributions that do not belong to the exponential families, and Section 4.5 studies the tightness of those bounds. Finally, Section 5 concludes this work. Appendix $\mathrm{A}$ recalls the Bayes error formula when class-conditional distributions belong to the univariate or the multivariate Gaussian families. 


\section{Bayes error and the total variation distance: An identity}

Recall Bayes error expression of Eq. 19.

$$
\begin{aligned}
B_{e} & =\int p(x) \min \left(r_{1}(x), r_{2}(x)\right) \mathrm{d} x \\
& =\int p(x) \min \left(c_{11} q_{1}(x)+c_{12} q_{2}(x), c_{21} q_{1}(x)+c_{22} q_{2}(x)\right) \mathrm{d} x
\end{aligned}
$$

with $q_{1}(x)=\frac{w_{1} p_{1}(x)}{p(x)}$ and $q_{2}(x)=\frac{w_{2} p_{2}(x)}{p(x)}$ the a posteriori probabilities. Using the mathematical rewriting trick:

$$
\min (a, b)=\frac{a+b}{2}-\frac{1}{2}|b-a|
$$

we get:

$$
B_{e}=\frac{1}{2} \int\left(a_{1} p_{1}(x)+a_{2} p_{2}(x)-\left|a_{2} p_{2}(x)-a_{1} p_{1}(x)\right|\right) \mathrm{d} x,
$$

where $a_{1}=w_{1}\left(c_{11}+c_{21}\right)$ and $a_{2}=w_{2}\left(c_{12}+c_{22}\right)$. Finally, using the fact that $\int p_{1}(x) \mathrm{d} x=\int p_{2}(x) \mathrm{d} x=1$, we end up with:

$$
B_{e}=\frac{a_{1}+a_{2}}{2}-\mathrm{TV}\left(a_{1} p_{1}, a_{2} p_{2}\right)
$$

where

$$
\mathrm{TV}(p, q)=\frac{1}{2} \int|p(x)-q(x)| \mathrm{d} x,
$$

denotes the total variation distance extended to positive distributions (i.e., not necessarily normalized probability distributions). In particular, for the probability of error, we have $a_{1}=w_{1}$ and $a_{2}=w_{2}$ (with $a_{1}+a_{2}=1$ ) and get:

$$
P_{e}=\frac{1}{2}-\mathrm{TV}\left(w_{1} p_{1}, w_{2} p_{2}\right)
$$

Note that $\operatorname{TV}\left(w_{1} p_{1}, w_{2} p_{2}\right)=w_{1} \operatorname{TV}\left(p_{1}, \frac{w_{2}}{w_{1}} p_{2}\right)=w_{2} \operatorname{TV}\left(\frac{w_{1}}{w_{2}} p_{1}, p_{2}\right)$. Therefore in the special case $w_{1}=w_{2}=\frac{1}{2}$, we get the probability of error related to the total variation distance (a metric) on probability distributions by:

$$
P_{e}=\frac{1}{2}\left(1-\mathrm{TV}\left(p_{1}, p_{2}\right)\right)
$$

For sanity check, notice that when $p_{1}=p_{2}$ (undistinguishable distributions), we have $\operatorname{TV}\left(p_{1}, p_{2}\right)=0$ and $P_{e}=\frac{1}{2}$. Clearly, $0 \leq \mathrm{TV}\left(p_{1}, p_{2}\right) \leq 1$ and $0 \leq P_{e} \leq \frac{1}{2}$. We summarize the result in the following theorem:

Theorem 1 The Bayes error $B_{e}$ for the cost design matrix $C=\left[c_{i j}\right]$ is related to the total variation metric distance $\operatorname{TV}(p, q)=\frac{1}{2} \int|p(x)-q(x)| \mathrm{d} x$ by $B_{e}=\frac{a_{1}+a_{2}}{2}-\mathrm{TV}\left(a_{1} p_{1}, a_{2} p_{2}\right)$ with $a_{1}=w_{1}\left(c_{11}+c_{21}\right)$ and $a_{2}=w_{2}\left(c_{12}+c_{22}\right)$. The identity simplifies for probability of error $P_{e}$ to $P_{e}=\frac{1}{2}-\mathrm{TV}\left(w_{1} p_{1}, w_{2} p_{2}\right)$.

Thus if we can compute the total variation distance of class-conditional probabilities $p_{1}$ and $p_{2}$, we can deduce the probability of error, and vice-versa:

$$
\operatorname{TV}\left(p_{1}, p_{2}\right)=1-2 P_{e} \geq 0
$$




\section{$3 \quad$ Upper bounds with generalized means}

Without loss of generality, consider the probability of error $P_{e}$ :

$$
P_{e}=\int \min \left(w_{1} p_{1}(x), w_{2} p_{2}(x)\right) \mathrm{d} x=S\left(w_{1} p_{1}, w_{2} p_{2}\right) .
$$

The probability of error can be interpreted as a similarity measure $S\left(w_{1} p_{1}, w_{2} p_{2}\right)$, extending the definition of histogram intersection [20] to continuous domains.

Chernoff [4] made use of the following mathematical trick:

$$
\min (a, b) \leq a^{\alpha} b^{1-\alpha}, \forall a, b>0
$$

to define the Chernoff information upper bounding $P_{e}$ :

$$
P_{e} \leq w_{1}^{\alpha^{*}} w_{2}^{1-\alpha^{*}} \rho_{*}\left(p_{1}, p_{2}\right)
$$

with

$$
\rho_{*}\left(p_{1}, p_{2}\right)=\min _{\alpha \in[0,1]} \rho_{\alpha}\left(p_{1}, p_{2}\right), \quad \rho_{\alpha}\left(p_{1}, p_{2}\right)=\int\left(p_{1}(x)\right)^{\alpha}\left(p_{2}(x)\right)^{1-\alpha} \mathrm{d} x .
$$

We shall revisit this technique using the wider scope of generalized weighted means.

By definition, a mean $M(a, b)$ is a smooth function such that $\min (a, b) \leq M(a, b) \leq \max (a, b)$. Similarly, we can define a weighted mean as a smooth function $M(a, b ; \alpha)$ that fulfills the interness property $M(a, b ; \alpha) \in$ $[\min (a, b), \max (a, b)] \forall \alpha \in[0,1]$. Let us consider the quasi-arithmetic means (also called Kolmogorov-Nagumo $f$-means [12, 14]) for a strictly monotonous generator function $f$ :

Lemma 1 ([1] $)$ The quasi-arithmetic weighted mean $M_{f}(a, b ; \alpha)=f^{-1}(\alpha f(a)+(1-\alpha) f(b))$ of two real values $a$ and $b$ for a strictly monotonic function $f$ satisfies the interness property: $\min (a, b) \leq M_{f}(a, b ; \alpha) \leq$ $\max (a, b)$.

Proof Assume $f$ is strictly increasing and $a \leq b$, then $f(a) \leq f(b)$ and $f(a) \leq \alpha f(a)+(1-\alpha) f(b) \leq f(b)$. Thus $a \leq M_{f}(a, b ; \alpha) \leq b$. If $b \leq a$, we similarly have $b \leq M_{f}(a, b ; \alpha) \leq a$. Therefore $\min (a, b) \leq$ $M_{f}(a, b ; \alpha) \leq \max (a, b)$. The proof is identical when $f$ is strictly decreasing.

Interestingly, one important property of quasi-arithmetic means is their dominance relationship. That is, if $f(x) \leq g(x)$ then $M_{f}(a, b ; \alpha) \leq M_{g}(a, b ; \alpha)$ (with equality when $\left.a=b\right)$. This property generalizes the well known arithmetic-geometric-harmonic (AGH) inequality property of Pythagorean means:

$$
M_{f_{a}}(a, b ; \alpha) \geq M_{f_{g}}(a, b ; \alpha) \geq M_{f_{h}}(a, b ; \alpha),
$$

with $f_{a}(x)=x, f_{g}(x)=\log x$ and $h(x)=1 / x$ denoting the generators for the arithmetic, geometric, and harmonic means, respectively. That is, we have:

$$
\alpha a+(1-\alpha) b \geq a^{\alpha} b^{1-\alpha} \geq \frac{a b}{\alpha a+(1-\alpha) b} .
$$

Similarly to Chernoff [4, we define the following generalized affinity coefficient $\rho_{f}$ using generalized weighted means as follows:

Definition 1 The Chernoff-type similarity coefficient (affinity) for a strictly monotonous function $f$ is defined by:

$$
\rho_{*}^{f}\left(p_{1}, p_{2}\right)=\min _{\alpha \in[0,1]} \int M_{f}\left(p_{1}(x), p_{2}(x) ; \alpha\right) \mathrm{d} x \leq \int p_{1}(x) \mathrm{d} x=1,
$$

and define the generalized Chernoff information as: 
Definition 2 The Chernoff-type information for a strictly monotonous function $f$ is defined by:

$$
C_{f}\left(p_{1}, p_{2}\right)=-\log \rho_{*}^{f}\left(p_{1}, p_{2}\right)=\max _{\alpha \in[0,1]}-\log \int M_{f}\left(p_{1}(x), p_{2}(x) ; \alpha\right) \mathrm{d} x \geq 0 .
$$

Corollary 1 The traditional Chernoff similarity, information, and upper bound are obtained by choosing the weighted geometric mean, by setting the generator $f_{\text {Chernoff }}(x)=\log (x)$ (with $f_{\text {Chernoff }}^{-1}(x)=\exp (x)$ ). We get $M_{f_{\text {Chernoff }}}\left(p_{1}(x), p_{2}(x) ; \alpha\right)=p_{1}(x)^{\alpha} p_{2}(x)^{1-\alpha}$.

When we do not optimize over the parameter $\alpha$, but assume it fixed to $\frac{1}{2}$, we extend the Bhattacharyya coefficient and divergence as follows:

Definition 3 The generalized skew Bhattacharyya-type similarity coefficient (affinity) for a strictly monotonous function $f$ is defined by:

$$
\rho_{\alpha}^{f}\left(p_{1}, p_{2}\right)=\int M_{f}\left(p_{1}(x), p_{2}(x) ; \alpha\right) \mathrm{d} x \leq \int p_{1}(x) \mathrm{d} x=1,
$$

and the generalized skew Bhattacharyya-type divergence is defined as $B_{\alpha}^{f}=-\log \rho_{\alpha}^{f}\left(p_{1}, p_{2}\right)$. The generalized Bhattacharyya coefficient $\rho^{f}\left(p_{1}, p_{2}\right)=\int M_{f}\left(p_{1}(x), p_{2}(x) ; \frac{1}{2}\right) \mathrm{d} x$ and divergence $B^{f}\left(p_{1}, p_{2}\right)=-\log \rho^{f}\left(p_{1}, p_{2}\right)$.

Theorem 2 Using quasi-arithmetic means, we can bound the probability of error as follows:

$$
P_{e}=\int \min \left(w_{1} p_{1}(x), w_{2} p_{2}(x)\right) \mathrm{d} x \leq \int M_{f}\left(w_{1} p_{1}(x), w_{2} p_{2}(x) ; \alpha\right) \mathrm{d} x .
$$

The upper bound proves useful for well-chosen $f$ yielding closed-form expression of the rhs.

In particular, by choosing the power means $M_{f_{\beta}}$ obtained for $f_{\beta}(x)=x^{\beta}$, we get a tight bound in the limit case since $M_{f_{\beta}}(p, q) \rightarrow \min (p, q)$ when $\beta \rightarrow-\infty$. However, in order for the generalized affinity, distance and upper bound to be useful, we need to be able to compute them in closed-form for some statistical distribution families. We illustrate how to derive closed form formula for $\rho_{f}$ and closed form upper bounds on $P_{e}$ for several statistical distribution families.

The generalized Bhattacharyya upper bound $\rho_{\frac{1}{2}}^{f}$ is obtained by setting $\alpha=\frac{1}{2}$ :

$$
\begin{aligned}
P_{e} & =\int \min \left(w_{1} p_{1}(x), w_{2} p_{2}(x)\right) \mathrm{d} x \leq B\left(w_{1} p_{1}(x), w_{2} p_{2}(x)\right), \\
B_{f}\left(w_{1} p_{1}(x), w_{2} p_{2}(x)\right) & =\int M_{f}\left(w_{1} p_{1}(x), w_{2} p_{2}(x) ; \frac{1}{2}\right) \mathrm{d} x .
\end{aligned}
$$

In order for the Chernoff information to improve over the Bhattacharyya bound, we need the quasiarithmetic $\alpha$-weighted mean to be a convex function with respect to parameter $\alpha$. For the geometric mean, we check that:

$$
M_{f_{g}}(a, b ; \alpha)=e^{\alpha \log \frac{a}{b}+\log b},
$$

is strictly convex with respect to $\alpha$ (since $\frac{\mathrm{d}^{2}}{\mathrm{~d} \alpha^{2}} M_{f_{g}}(a, b ; \alpha)=\left(\log \frac{a}{b}\right)^{2} M_{f_{g}}(a, b ; \alpha)>0$ for $\left.a \neq b\right)$. Similarly, the weighted harmonic mean is convex with respect to $\alpha$ since $\frac{\mathrm{d}^{2}}{\mathrm{~d} \alpha^{2}} M_{f_{h}}(a, b ; \alpha)=2 a b(a-b)^{2}(\alpha(a-b)+b)^{-3}>0$.

Remark 2 Note that not all quasi-arithmetic means yield convex weighted means. Indeed, let $M_{f}^{\prime}(a, b ; \alpha)=$ $f\left(\alpha\left(f^{-1}(a)-f^{-1}(b)\right)+f^{-1}(b)\right)$ then $\frac{\mathrm{d}^{2}}{\mathrm{~d} \alpha^{2}} M_{f}^{\prime}(a, b ; \alpha)=\left(f^{-1}(a)-f^{-1}(b)\right)^{2} f^{\prime \prime}\left(\alpha\left(f^{-1}(a)-f^{-1}(b)\right)+f^{-1}(b)\right)$. Functions $f$ and $f^{-1}$ are strictly monotonous but can be convex, concave, or arbitrary in general.

Remark 3 Sometimes, we prefer to parameterize the weighted mean as the smooth interpolant from a to b, when $\alpha$ varies from 0 to 1 (kind of geodesic parameterization). In that case, we may prefer the parameterization $M_{f}\left(a, b ; \alpha^{\prime}\right)=f^{-1}\left(\left(1-\alpha^{\prime}\right) f(a)+\alpha^{\prime} f(b)\right)$. This is not important for Bhattacharyya-type symmetric bounds nor for Chernoff-type bounds that optimize over the $\alpha$ range (or equivalently over the $\alpha^{\prime}$ range).

Let us now examine how the "quasi-arithmetic bounding techniques" apply for several families of statistical distributions. 


\section{Some illustrating examples}

\subsection{Geometric means and the Chernoff bound for exponential families}

First, we recall the well-known formula [11, 16] for the case of exponential families.

In order to compute a closed form for the right-hand side of Eq. 58 ;

$$
\begin{aligned}
P_{e} & \leq \int M_{f}\left(w_{1} p_{1}(x), w_{2} p_{2}(x) ; \alpha\right) \mathrm{d} x \\
& \leq \int f^{-1}\left(\alpha f\left(w_{1} p_{1}\right)+(1-\alpha) f\left(w_{2} p_{2}\right)\right) \mathrm{d} x
\end{aligned}
$$

we consider the geometric mean obtained for $f(x)=\log x$. Since $p_{1}(x)=\exp \left(x^{\top} \theta_{1}-F\left(\theta_{1}\right)\right)$ and $p_{2}(x)=$ $\exp \left(x^{\top} \theta_{2}-F\left(\theta_{2}\right)\right)$ belong to the exponential families, we get:

$$
\begin{aligned}
M_{f}\left(w_{1} p_{1}(x), w_{2} p_{2}(x) ; \alpha\right) & =e^{\alpha \log w_{1} p_{1}(x)+(1-\alpha) \log w_{2} p_{2}(x)} \\
& =w_{1}^{\alpha} w_{2}^{1-\alpha} p_{1}^{\alpha}(x) p_{2}^{1-\alpha}(x)
\end{aligned}
$$

It follows that:

$$
P_{e} \leq w_{1}^{\alpha} w_{2}^{1-\alpha} \int f^{-1}\left(\alpha f\left(p_{1}(x)\right)+(1-\alpha) f\left(p_{2}(x)\right)\right) \mathrm{d} x
$$

Remark 4 In fact, the geometric mean is a limit case of a family of linear-scale free means defined for $f_{\alpha}(x)=x^{\frac{1-\alpha}{2}}$ with $f_{1}(x)=\log x$. In general, in order to slide the a priori weights $w_{1}$ and $w_{2}$ out of the integral, we would like to use a homogeneous function $f($ with $f(\lambda x)=g(\lambda) f(x))$.

Furthermore, since $m_{\alpha}\left(x ; \theta_{1}, \theta_{2}\right)=\alpha \log \left(p_{1}(x)\right)+(1-\alpha) \log \left(p_{2}(x)\right)=x^{\top}\left(\alpha \theta_{1}+(1-\alpha) \theta_{2}\right)-\alpha F\left(\theta_{1}\right)-$ $(1-\alpha) F\left(\theta_{2}\right)$, we would like to get $f^{-1}\left(m_{\alpha}\left(x ; \theta_{1}, \theta_{2}\right)\right)$ as $c_{\theta_{1}, \theta_{2} ; \alpha} p\left(x ; \theta_{12}^{(\alpha)}\right)$ so that we can slide the integral operand inside the expression, and use the fact that we recognize a member $\theta_{12}^{(\alpha)}$ of the exponential family so that its integration over the support is 1 . For members of the same exponential family, we have:

$$
\begin{aligned}
f^{-1}\left(m_{\alpha}\left(x ; \theta_{1}, \theta_{2}\right)\right) & =e^{F\left(\alpha \theta_{1}+(1-\alpha) \theta_{2}\right)-\alpha F\left(\theta_{1}\right)-(1-\alpha) F\left(\theta_{2}\right)} p\left(x ; \alpha \theta_{1}+(1-\alpha) \theta_{2}\right), \\
& =e^{-J_{F}^{(\alpha)}\left(\theta_{1}, \theta_{2}\right)} p(x ; \underbrace{\alpha \theta_{1}+(1-\alpha) \theta_{2}}_{\theta_{12}^{(\alpha)}})
\end{aligned}
$$

Thus

$$
P_{e} \leq w_{1}^{\alpha} w_{2}^{1-\alpha} e^{-J_{F}^{(\alpha)}\left(\theta_{1}, \theta_{2}\right)} \int p\left(x ; \alpha \theta_{1}+(1-\alpha) \theta_{2}\right) \mathrm{d} x
$$

Since the natural parameter space $\Theta$ is convex for exponential families, we have $\theta_{12}^{(\alpha)}=\alpha \theta_{1}+(1-\alpha) \theta_{2} \in \Theta$ and therefore $\int p\left(x ; \alpha \theta_{1}+(1-\alpha) \theta_{2}\right) \mathrm{d} x=1$. We end up with:

$$
P_{e} \leq \min _{\alpha \in[0,1]} w_{1}^{\alpha} w_{2}^{1-\alpha} e^{-J_{F}^{(\alpha)}\left(\theta_{1}, \theta_{2}\right)}
$$

Definition 4 The $\alpha$-Chernoff distance is an asymmetric statistical distance defined by $\rho_{\alpha}\left(p_{1}, p_{2}\right)=$ $-\log p_{1}^{\alpha}(x) p_{2}^{1-\alpha}(x) \mathrm{d} x \geq 0$. The Bhattacharrya symmetric distance $\rho_{\frac{1}{2}}\left(p_{1}, p_{2}\right)$ is a particular member of the family of $\alpha$-Chernoff distances. 
Thus we always have $\rho_{\frac{1}{2}}\left(p_{1}, p_{2}\right) \geq \rho_{*}\left(p_{1}, p_{2}\right)=\min _{\alpha \in[0,1]} \rho_{\alpha}\left(p_{1}, p_{2}\right)$.

The optimal Chernoff bound is obtained for the optimized weight $\alpha^{*}$ that has been characterized geometrically on the statistical manifold [15].

To derive other Chernoff-type upper bounds, we shall therefore consider non-exponential families of distributions. The most prominent family, to start with, is the Cauchy family (a member of the Student $t$-distribution families) and the multivariate Pearson type VII elliptical distributions [19] (with its scaled multivariate $t$-distributions).

\subsection{Harmonic means and the Chernoff-type bound for Cauchy distributions}

Consider the family of Cauchy distributions with density:

$$
p(x ; s)=\frac{1}{\pi} \frac{s}{x^{2}+s^{2}},
$$

defined over the support $\mathbb{R}$. This is a scale family with heavy tails indexed by a scale parameter $s$ :

$$
p(x ; s)=\frac{1}{s} p_{0}\left(\frac{x}{s}\right), \quad p_{0}(x)=\frac{1}{\pi} \frac{1}{x^{2}+1},
$$

where $p_{0}(x)$ denotes the standard Cauchy distribution $C_{0}$. Cauchy distributions do not belong to the exponential families. (Indeed, the mean is undefined.) Let us take the harmonic mean $M_{H}=M_{f}$ defined for the strictly monotonous generator $f(x)=f^{-1}(x)=\frac{1}{x}$. We have:

$$
P_{e}=\int \min \left(w_{1} p_{1}(x), w_{2} p_{2}(x)\right) \mathrm{d} x \leq M_{f}\left(w_{1} p_{1}(x), w_{2} p_{2}(x) ; \alpha\right) \mathrm{d} x .
$$

Wlog., to simplify calculations exhibiting the method, consider $w_{1}=w_{2}=\frac{1}{2}$.

$$
\begin{aligned}
& P_{e} \leq \int M_{H}\left(\frac{1}{2} p_{1}(x), \frac{1}{2} p_{2}(x) ; \alpha\right) \mathrm{d} x, \\
& \leq \frac{1}{2} \int \frac{p_{1}(x) p_{2}(x)}{(1-\alpha) p_{1}(x)+\alpha p_{2}(x)} \mathrm{d} x, \\
& \leq \frac{1}{2} \int \frac{\frac{s_{1}}{\pi\left(x^{2}+s_{1}^{2}\right)} \frac{s_{2}}{\pi\left(x^{2}+s_{2}^{2}\right)}}{(1-\alpha) \frac{s_{1}}{\pi\left(x^{2}+s_{1}^{2}\right)}+\alpha \frac{s_{2}}{\pi\left(x^{2}+s_{2}^{2}\right)}} \mathrm{d} x, \\
& \leq \frac{1}{2} \int \frac{s_{1} s_{2}}{\pi\left((1-\alpha) s_{1}\left(x^{2}+s_{2}^{2}\right)+\alpha s_{2}\left(x^{2}+s_{1}^{2}\right)\right)} \mathrm{d} x, \\
& \leq \frac{1}{2} \int \frac{s_{1} s_{2}}{\pi\left(\left((1-\alpha) s_{1}+\alpha s_{2}\right) x^{2}+(1-\alpha) s_{1} s_{2}^{2}+\alpha s_{2} s_{1}^{2}\right)} \mathrm{d} x, \\
& \leq \frac{1}{2} \frac{s_{1} s_{2}}{\left((1-\alpha) s_{1}+\alpha s_{2}\right) s_{\alpha}} \underbrace{\int \frac{1}{\pi} \frac{s_{\alpha}}{x^{2}+s_{\alpha}^{2}} \mathrm{~d} x}_{=1},
\end{aligned}
$$

since $s_{\alpha}>0$ belongs to the parameter space $\Theta$, with:

$$
s_{\alpha}=\sqrt{\frac{(1-\alpha) s_{1} s_{2}^{2}+\alpha s_{2} s_{1}^{2}}{(1-\alpha) s_{1}+\alpha s_{2}}} .
$$

Thus the weighted harmonic mean provides a Chernoff-type upper bound:

$$
P_{e} \leq \frac{1}{2} \frac{s_{1} s_{2}}{\left((1-\alpha) s_{1}+\alpha s_{2}\right) \sqrt{\frac{(1-\alpha) s_{1} s_{2}^{2}+\alpha s_{2} s_{1}^{2}}{(1-\alpha) s_{1}+\alpha s_{2}}}} .
$$


A sanity check $s_{1}=s_{2}=s$ shows that $P_{e}=\frac{1}{2}$, as expected (class-conditional distributions are not distinguishable).

The Bhattacharrya-type bound obtained for $\alpha=\frac{1}{2}$ yields:

$$
s_{\frac{1}{2}}=\sqrt{\frac{s_{1} s_{2}^{2}+s_{2} s_{1}^{2}}{s_{1}+s_{2}}},
$$

and the upper bound:

$$
P_{e} \leq \frac{s_{1} s_{2}}{\sqrt{\left(s_{1}+s_{2}\right)\left(s_{1} s_{2}^{2}+s_{2} s_{1}^{2}\right)}} .
$$

The harmonic mean $M_{H}(a, b ; \alpha)$ is linear-scale free: $M_{H}(\lambda a, \lambda b ; \alpha)=\lambda M_{H}(a, b ; \alpha)$. Let $\lambda=\frac{s_{2}}{s_{1}}$. Then we write the probability of error as:

$$
P_{e} \leq \frac{1}{2} \frac{\lambda}{\sqrt{(1-\alpha+\alpha \lambda)\left((1-\alpha) \lambda^{2}+\alpha \lambda\right)}}=P_{e}^{(\alpha)} .
$$

In particular, we upper bound the probability of error by the following Bhattacharyya bound:

$$
P_{e} \leq P_{e}^{\left(\frac{1}{2}\right)}=\frac{\sqrt{\lambda}}{\lambda+1}
$$

The Chernoff-type bound proceeds by minimizing Eq. 80 with respect to $\alpha$. We have $P_{e}^{(0)}=P_{e}^{(1)}=\frac{1}{2}$. To find the minimum value over the $\alpha$-range [0,1], we study function $P_{e}^{(\alpha)}$. Using a computer-algebra system 3 , we find that it is a convex function that always admits a minimum at $\alpha=\frac{1}{2}$. Indeed, the derivative of $P_{e}$ with respect to $\alpha$ is:

$$
\frac{\mathrm{d}}{\mathrm{d} \alpha} P_{e}(\alpha ; \lambda)=\frac{\frac{1}{4}(\lambda-1)^{2} \lambda^{2}(2 \alpha-1)}{(\lambda((\lambda-1) \alpha+1)(-\alpha \lambda+\lambda+\alpha))^{3 / 2}},
$$

that is zero if and only if $\alpha=\frac{1}{2}$. This is a remarkable example that shows that the Chernoff bound amounts to the Bhatthacharrya bound.

Let us compute the total variation distance between two scaled Cauchy distributions $a_{1} p\left(x ; s_{1}\right)$ and $a_{2} p\left(x ; s_{2}\right)$ defined over the real-line support $\mathbb{R}$ with:

$$
p(x ; s)=\frac{1}{\pi} \frac{s}{x^{2}+s^{2}}
$$

For $s_{1} \neq s_{2}$, the two distinct positive densities intersect in exactly two values of the support:

$$
\begin{aligned}
& x_{1}=-\frac{\sqrt{s_{1} s_{2}\left(a_{2} s_{1}-a_{1} s_{2}\right)}}{\sqrt{a_{1} s_{1}-a_{2} s_{2}}}, \\
& x_{2}=\frac{\sqrt{s_{1} s_{2}\left(a_{2} s_{1}-a_{1} s_{2}\right)}}{\sqrt{a_{1} s_{1}-a_{2} s_{2}}}
\end{aligned}
$$

In particular, when $a_{1}=a_{2}=1$ (ie., probability densities), we have $x_{1}=-\sqrt{s_{1} s_{2}}$ and $x_{2}=\sqrt{s_{1} s_{2}}$. By abuse of notations, we let $x_{0}=-\infty$ and $x_{3}=\infty$, and apply the generic 1D total variation formula of Eq. 114 with $k=2$ :

$$
\operatorname{TV}\left(a_{1} p_{1}, a_{2} p_{2}\right)=\frac{1}{2} \sum_{i=1}^{k+1}\left|\left(P_{1}\left(x_{i}\right)+P_{2}\left(x_{i+1}\right)-P_{1}\left(x_{i-1}\right)-P_{2}\left(x_{i}\right)\right)\right| .
$$

\footnotetext{
${ }^{3}$ Namely, Wolfram Alpha online, http://www.wolframalpha.com
} 
Since the Cauchy scaled cumulative distribution is $P_{i}(x)=a_{i}\left(\frac{1}{\pi} \arctan \left(\frac{x}{s_{i}}\right)+\frac{1}{2}\right)$ with $P_{i}\left(x_{0}\right)=0$ and $P_{i}\left(x_{3}\right)=a_{i}$.

In particular, the probability of error when $w_{1}=w_{2}=\frac{1}{2}$ is:

$$
\begin{aligned}
P_{e} & =\frac{1}{2}\left(1-\mathrm{TV}\left(p_{1}, p_{2}\right)\right), \\
\mathrm{TV}\left(p_{1}, p_{2}\right) & =\left|P_{1}\left(\sqrt{s_{1} s_{2}}\right)-P_{1}\left(-\sqrt{s_{1} s_{2}}\right)-P_{2}\left(\sqrt{s_{1} s_{2}}\right)+P_{2}\left(-\sqrt{s_{1} s_{2}}\right)\right| .
\end{aligned}
$$

That is, we find an exact analytic expression for the total variation (and hence for Bayes error $B_{e}$ and the probability of error $P_{e}$ ):

$$
\begin{gathered}
\operatorname{TV}\left(p_{1}, p_{2}\right)=\frac{1}{\pi}\left(\arctan \left(\sqrt{\frac{s_{2}}{s_{1}}}\right)-\arctan \left(-\sqrt{\frac{s_{2}}{s_{1}}}\right)\right. \\
\left.+\arctan \left(-\sqrt{\frac{s_{1}}{s_{2}}}\right)-\arctan \left(\sqrt{\frac{s_{1}}{s_{2}}}\right)\right) .
\end{gathered}
$$

Using the identity $\arctan (x)+\arctan (1 / x)=\frac{\pi}{2}$ and the fact that $\arctan (-x)=-\arctan (x)$, we get a more compact formula:

$$
\operatorname{TV}\left(p_{1}, p_{2}\right)=\frac{2}{\pi}\left(\arctan \left(\sqrt{\frac{s_{2}}{s_{1}}}\right)-\arctan \left(\sqrt{\frac{s_{1}}{s_{2}}}\right)\right) .
$$

Remark 5 Note that for Cauchy distributions with scale parameter $s_{1}$ and $s_{2}$, we have $\operatorname{TV}\left(s_{1}, s_{2}\right)=$ $\operatorname{TV}\left(\lambda s_{1}, \lambda s_{2}\right), \forall \lambda>0$ (because $\sqrt{\frac{\lambda s_{1}}{\lambda s_{2}}}=\sqrt{\frac{s_{1}}{s_{2}}}$ ). Therefore, we may renormalize by considering $s_{1} \leftarrow 1$ and $s_{2} \leftarrow \frac{s_{2}}{s_{1}}$.

It follows that the probability of error is:

$$
\begin{aligned}
P_{e} & =\frac{1}{2}-\frac{1}{\pi}(\arctan (\sqrt{\lambda})-\arctan (\sqrt{1 / \lambda})), \\
& =1-\frac{2}{\pi} \arctan (\sqrt{\lambda}), \quad \lambda=\frac{s_{2}}{s_{1}} .
\end{aligned}
$$

Consider the following numerical example: $s_{1}=10$ and $s_{2}=50\left(w_{1}=w_{2}=\frac{1}{2}\right)$. Then, we get $P_{e} \sim 0.2677$, the Bhattacharyya-type bound $B \sim 0.3726$ and the Chernoff-type bound $C=B$.

Remark 6 Let us study the tightness of the upper bound. We can express analytically the gap as $\Delta=$ $C-P_{e}=C-\frac{1}{2}+\mathrm{TV}\left(p_{1}, p_{2}\right)$. That is, we get:

$$
\Delta(\lambda)=\frac{\sqrt{\lambda}}{1+\lambda}-1+\frac{2}{\pi} \arctan (\sqrt{\lambda})>0 .
$$

Note that $\lambda=1$, we have $\Delta(1)=0$ : That is, the gap is tight when distributions coincide. Using a computer-algebra system, we find that the gap is maximized at $\lambda=\frac{2+\pi}{\pi-2} \sim 4.5$ and $\Delta_{\max } \sim 0.1$.

This Cauchy case study illustrates well that the geometric mean may not always be the most appropriate weighted mean to use to upper bound $P_{e}$. Note that for Cauchy distributions, we also obtained an analytic form of $P_{e}$ and $B_{e}$ using the total variation distance expressed using the cumulative distribution.

We showed that the harmonic mean is tailored to derive closed-form bound for the Cauchy distributions. However, we may apply the harmonic mean to other distributions. This has in fact be done in the literature detailed in the following remark:

Remark 7 The harmonic bean $M_{H}(a, b)=\frac{2 a b}{(a+b)}$ has been used for defining the nearest neighbor error bound [7], always better than the Bhattacharrya bound (obtained for $\alpha=\frac{1}{2}$ ).

(Note that for exponential families, the nearest neighbor error bound is not available in closed-form.) 


\subsection{Pearson type VII distributions}

Consider the $d$-dimensional elliptically symmetric Pearson type VII distribution [19, 2]) with density:

$$
p(x ; \mu, \Sigma, \lambda)=\pi^{-\frac{d}{2}} \frac{\Gamma(\lambda)}{\Gamma\left(\lambda-\frac{d}{2}\right)}|\Sigma|^{-\frac{1}{2}}\left(1+(x-\mu)^{\top} \Sigma^{-1}(x-\mu)\right)^{-\lambda}
$$

where $\Gamma(z)=\int_{0}^{\infty} t^{z-1} e^{-t} \mathrm{~d} t$ denotes the Gamma function extending the factorial function (i.e., $\Gamma(1)=1$, $\Gamma(x+1)=x \Gamma(x)$ and $\Gamma(n)=(n-1)$ ! for $n \in \mathbb{N})$. Parameter $\lambda>\frac{d}{2}$ represents the degree of freedom [19].

For sake of simplicity, wlog., let us consider the zero-centered sub-family with $\mu=0, v=1$ and $\lambda>\frac{d}{2}$ fixed. This sub-family is defined on the cone of symmetric positive definite matrices $\Theta=\{\Sigma \mid \Sigma \succ 0\}$ with density:

$$
p(x ; \Sigma)=c_{d}(\lambda)|\Sigma|^{-\frac{1}{2}}\left(1+x^{\top} \Sigma^{-1} x\right)^{-\lambda},
$$

where $c_{d}(\lambda)=\pi^{-\frac{d}{2}} \frac{\Gamma(\lambda)}{\Gamma\left(\lambda-\frac{d}{2}\right)}$ is the normalizing constant [13]. This multivariate family does not belong to the exponential families. Consider the $\alpha$-weighted $f$-mean with $f(x)=x^{-\frac{1}{\lambda}}$, for prescribed $\lambda>\frac{d}{2}$ (and $\left.f^{-1}(x)=x^{-\lambda}\right)$.

We have:

$$
\begin{aligned}
\alpha f\left(p_{1}\right) & =\alpha c_{d}(\lambda)^{-1 / \lambda}\left|\Sigma_{1}\right|^{\frac{1}{2 \lambda}}\left(1+x^{\top} \Sigma_{1}^{-1} x\right), \\
(1-\alpha) f\left(p_{2}\right) & =(1-\alpha) c_{d}(\lambda)^{-1 / \lambda}\left|\Sigma_{2}\right|^{\frac{1}{2 \lambda}}\left(1+x^{\top} \Sigma_{1}^{-2} x\right) .
\end{aligned}
$$

Let $c_{1}=c_{d}(\lambda)^{-1 / \lambda}\left|\Sigma_{1}\right|^{\frac{1}{2 \lambda}}$ and $c_{2}=c_{d}(\lambda)^{-1 / \lambda}\left|\Sigma_{2}\right|^{\frac{1}{2 \lambda}}$. Denote by:

$$
\begin{aligned}
c_{\alpha} & =\alpha c_{1}+(1-\alpha) c_{2}, \\
& =c_{d}(\lambda)^{-1 / \lambda}\left(\alpha\left|\Sigma_{1}\right|^{\frac{1}{2 \lambda}}+(1-\alpha)\left|\Sigma_{2}\right|^{\frac{1}{2 \lambda}}\right),
\end{aligned}
$$

we get:

$$
\begin{aligned}
\alpha f\left(p_{1}\right)+(1-\alpha) f\left(p_{2}\right) & =\alpha c_{1}\left(1+x^{\top} \Sigma_{1}^{-1} x\right)+(1-\alpha) c_{2}\left(1+x^{\top} \Sigma_{2}^{-1} x\right) \\
& =c_{\alpha}\left(1+x^{\top} \Sigma_{\alpha}^{-1} x\right)
\end{aligned}
$$

with

$$
\begin{aligned}
\Sigma_{\alpha}^{-1} & =\frac{\alpha c_{1} \Sigma_{1}^{-1}+(1-\alpha) c_{2} \Sigma_{2}^{-1}}{c_{\alpha}}, \\
& =\frac{\alpha\left|\Sigma_{1}\right|^{\frac{1}{2 \lambda}} \Sigma_{1}^{-1}+(1-\alpha)\left|\Sigma_{2}\right|^{\frac{1}{2 \lambda}} \Sigma_{2}^{-1}}{\left(\alpha\left|\Sigma_{1}\right|^{\frac{1}{2 \lambda}}+(1-\alpha)\left|\Sigma_{2}\right|^{\frac{1}{2 \lambda}}\right)} \succ 0 .
\end{aligned}
$$

Therefore,

$$
\begin{aligned}
f^{-1}\left(\alpha f\left(p_{1}\right)+(1-\alpha) f\left(p_{2}\right)\right) & =c_{\alpha}^{-\lambda}\left(1+x^{\top} \Sigma_{\alpha}^{-1} x\right)^{-\lambda} \\
& =c_{\alpha}^{-\lambda}\left|\Sigma_{\alpha}\right|^{\frac{1}{2}} \frac{1}{c_{d}(\lambda)} p\left(x ; \Sigma_{\alpha}\right)
\end{aligned}
$$

It follows that: 


$$
\begin{aligned}
P_{e} & \leq \frac{1}{2}\left(\alpha\left|\Sigma_{1}\right|^{\frac{1}{2 \lambda}}+(1-\alpha)\left|\Sigma_{2}\right|^{\frac{1}{2 \lambda}}\right)^{-\lambda}\left|\Sigma_{\alpha}\right|^{\frac{1}{2}} \underbrace{\int p\left(x ; \Sigma_{\alpha}\right) \mathrm{d} x}_{=1} \\
& =\frac{1}{2}\left(\alpha\left|\Sigma_{1}\right|^{\frac{1}{2 \lambda}}+(1-\alpha)\left|\Sigma_{2}\right|^{\frac{1}{2 \lambda}}\right)^{-\lambda}\left|\Sigma_{\alpha}\right|^{\frac{1}{2}} .
\end{aligned}
$$

since $\Sigma_{\alpha} \in \Theta$.

The Pearson type VI distribution is related to the multivariate $t$-distributions [13].

\subsection{Central multivariate $t$-distributions}

The multivariate $t$-distribution (MVT, centered at $\mu=0$ ) with $\nu \geq 1$ degrees of freedom is defined for a positive definite matrix $\Sigma \succ 0$ (the scale matrix) by the following density:

$$
p(x ; \Sigma)=c_{d, \nu}|\Sigma|^{-\frac{1}{2}}\left(1+\frac{1}{\nu} x^{\top} \Sigma^{-1} x\right)^{-\frac{\nu+d}{2}},
$$

where $c_{d, \nu}=\frac{\Gamma\left(\frac{\nu+d}{2}\right)}{\Gamma\left(\frac{\nu}{2}\right)(\nu \pi)^{\frac{d}{2}}}$ is the constant normalizing the distribution. The covariance matrix is $\frac{\nu}{\nu-2} \Sigma$.

Let $t=-\frac{\nu+d}{2}$, and consider $f(x)=x^{\frac{1}{t}}$, with functional inverse $f^{-1}(x)=x^{t}$. Using a technique similar to the Pearson bound, after massaging the mathematics, we find that (for $\left.w_{1}=w_{2}=\frac{1}{2}\right) P_{e} \leq \frac{1}{2} \rho_{\alpha}^{\mathrm{MVT}}\left(\Sigma_{1}, \Sigma_{2}\right)$ (for $\alpha \in[0,1]$ ), with:

$$
\rho_{\alpha}^{\mathrm{MVT}}\left(\Sigma_{1}, \Sigma_{2}\right)=\left(\alpha\left|\Sigma_{1}\right|^{-\frac{1}{2 t}}+(1-\alpha)\left|\Sigma_{2}\right|^{-\frac{1}{2 t}}\right)^{t}\left|\Sigma_{\alpha}^{\prime}\right|^{\frac{1}{2}}
$$

and

$$
\Sigma_{\alpha}^{\prime}=\left(\frac{\alpha\left|\Sigma_{1}\right|^{-\frac{1}{2 t}} \Sigma_{1}^{-1}+(1-\alpha)\left|\Sigma_{2}\right|^{-\frac{1}{2 t}} \Sigma_{2}^{-1}}{\alpha\left|\Sigma_{1}\right|^{-\frac{1}{2 t}}+(1-\alpha)\left|\Sigma_{2}\right|^{-\frac{1}{2 t}}}\right)^{-1} .
$$

Note that when $\nu \rightarrow \infty$, and $t=-\frac{2}{\nu+d} \rightarrow 0$, the multivariate $t$-distribution (MVT) tend to a multivariate Normal distribution $(\mathrm{MVN})$ with covariance matrix $\Sigma$. The power mean induced by $f(x)=x^{\frac{1}{t}}$ (with $t=-\frac{\nu+d}{2}$ ) tends to the geometric mean, and we get the well-known Bhattacharyya coefficient bound (for $\alpha=\frac{1}{2}$ ) on central multivariate Gaussians [16] (see Eq. 35):

$$
P_{e} \leq \frac{1}{2} \rho^{\mathrm{MVN}}\left(\Sigma_{1}, \Sigma_{2}\right), \quad \rho^{\mathrm{MVN}}\left(\Sigma_{1}, \Sigma_{2}\right)=\frac{\left|\Sigma_{1}\right|^{\frac{1}{4}}\left|\Sigma_{2}\right|^{\frac{1}{4}}}{\left|\frac{1}{2} \Sigma_{1}+\frac{1}{2} \Sigma_{2}\right|^{\frac{1}{2}}} .
$$

\subsection{Assessing the Bhattacharyya-type and the Chernoff-type upper bounds}

Let us recall the inequality on the probability of error $P_{e}$ between two distributions $p_{1}$ and $p_{2}$ with equal prior $\left(w_{1}=w_{2}=\frac{1}{2}\right)$ :

$$
P_{e}\left(p_{1}, p_{2}\right)=\frac{1}{2}\left(1-\mathrm{TV}\left(p_{1}, p_{2}\right)\right) \leq \frac{1}{2} \rho_{*}^{f}\left(p_{1}, p_{2}\right) \leq \frac{1}{2} \rho^{f}\left(p_{1}, p_{2}\right) \leq \frac{1}{2} .
$$

The left hand side has been elucidated in Eq. 44 and the right hand side is the Chernofftype/Bhattacharyya-type similarity coefficients which should be available in closed-form for fast calculation. We are interested in characterizing the gaps $\Delta=\rho\left(p_{1}, p_{2}\right)-P_{e}$ and $\Delta_{*}=\rho_{*}\left(p_{1}, p_{2}\right)-P_{e}$ between the Bhattacharyya/Chernoff upper bounds and $P_{e}$ (with $\Delta_{*} \leq \Delta$ ). We start with some simple cases of univariate distributions, where $P_{e}$ can be expressed analytically, and then considered the multivariate distributions where $P_{e}$ need to be stochastically estimated. 


\subsubsection{Simple cases: Univariate distributions}

For univariate densities, we may calculate the total variation by computing the roots of $p_{1}(x)=p_{2}(x)$ and then using the cumulative distributions $P(t)=\int p(x \leq t) \mathrm{d} x$ to explicit a formula. Assume $x_{1}, \ldots, x_{k}$ are the $k$ roots, and by abuse of notations, let $x_{0}=x_{\min }$ and $x_{k+1}=x_{\max }$ denote the extra endpoints of the distribution support $\left(x \in\left[x_{\min }, x_{\max }\right]\right)$. When the support is the full real line $\left(\operatorname{supp}\left(p_{i}\right)=\mathbb{R}\right)$, we set $x_{\min }=-\infty$ and $x_{\max }=\infty$. We have:

$$
\begin{aligned}
\operatorname{TV}\left(p_{1}, p_{2}\right) & =\frac{1}{2} \sum_{i=1}^{k+1}\left|\int_{x_{i-1}}^{x_{i}}\left(p_{1}(x)-p_{2}(x)\right) \mathrm{d} x\right| \\
& =\frac{1}{2}\left|\sum_{i=1}^{k+1}\left(P_{1}\left(x_{i}\right)+P_{2}\left(x_{i+1}\right)-P_{1}\left(x_{i-1}\right)-P_{2}\left(x_{i}\right)\right)\right| .
\end{aligned}
$$

This scheme allows one to compute the total variation distance of many univariate distributions like the Gaussian (see Eq. 130), Rayleigh, Cauchy, etc distributions. Therefore, we get analytic expressions of Bayes error relying on the cumulative distribution function (CDF) for many univariate distributions.

Those the Bhattacharyya gaps for the Cauchy or 1D Gaussians can be written analytically. Section 4.2 already addressed the gap for the Cauchy distributions. Next, we consider the general multivariate case (that includes those univariate examples) and report on our numerical experiments.

\subsubsection{The case of multivariate distributions}

We consider multivariate $t$-distributions [8, 9] (MVT) that includes the multivariate normal (MVN) distributions in the limit case (when the number of degree of freedom tends to infinity). To perform experiments, we used the mvtnorm packag 4 on the $\mathrm{R}$ software platform 5

Since the total variation (TV) does not admit a closed-form formula (nor the probability of error $P_{e}$ ), we estimate those quantities by performing stochastic integrations as follows:

$$
\begin{aligned}
\widehat{P_{e}}\left(p_{1}, p_{2}\right) & =\frac{1}{2}\left(1-\widehat{\mathrm{TV}}\left(p_{1}, p_{2}\right)\right) \\
\widehat{\mathrm{TV}}\left(p_{1}, p_{2}\right) & =\frac{1}{2 n} \sum_{i=1}^{n} \frac{1}{p_{1}(x)}\left|p_{1}(x)-p_{2}(x)\right| \\
& =\frac{1}{2 n} \sum_{i=1}^{n}\left|1-\frac{p_{2}\left(x_{i}\right)}{p_{1}\left(x_{i}\right)}\right|
\end{aligned}
$$

where $x_{1}, \ldots, x_{n}$ are $n$ identically and independently variates of $p_{1}$. Stochastic integration guarantees convergence to the true value in the $\operatorname{limit:}_{\lim } \rightarrow \infty \widehat{P}_{e}\left(p_{1}, p_{2}\right)=P_{e}\left(p_{1}, p_{2}\right)$.

To give a numerical example, consider central bidimensional $t$-distributions with $\nu=6$ and scale matrices $\Sigma_{1}=I$ and $\Sigma_{2}=10 I$, where $I$ denotes the identity matrix. Running the mvtTotalVariation (df , sigma1, sigma2, n) code (see Appendix), we get the following estimates for $\widehat{T V}$ : $0.3210709(n=100), 0.3479519(n=1000), 0.3472926(n=10000), 0.347538(n=100000)$. We chose $n=10000$ in the following experiments.

Consider central $t$-distributions $(\mu=0)$. We implemented the closed-form formula of Eq. 109 to calculate the $\alpha$-Chernoff coefficient $\rho_{\alpha}^{\mathrm{MVT}}\left(\Sigma_{1}, \Sigma_{2}\right)$. The optimal Chernoff coefficient (and the exponent $\alpha^{*}$ ) is approximated by discretizing into 1000 steps the unit range for $\alpha$. We consider $\Sigma_{1}=I$ and $\Sigma_{2}=\lambda I$ (with $\nu=6$ ) for $\lambda=d+1$ and various values of the dimension. (Indeed, after an appropriate "whitening" transformation [7],

\footnotetext{
${ }^{4}$ http://cran.r-project.org/web/packages/mvtnorm/index.html To install the package, we used the command line: install.packages ('mvtnorm_0.9-9996.zip', repos $=$ NULL)

${ }^{5} \mathrm{R}$ can be freely downloaded at http://www.r-project.org/
} 
Table 1: Experimental results for central multivariate $t$-distributions (MVT) with $\nu=6, \Sigma_{1}=I$ and $\Sigma_{2}=\lambda I$. We have: $\widehat{P_{e}} \leq \frac{1}{2} \rho_{*}^{\mathrm{MVT}} \leq \frac{1}{2} \rho^{\mathrm{MVT}}$ where $\rho_{*}^{\mathrm{MVT}}$ and $\rho^{\mathrm{MVT}}$ denotes the Chernoff and Bhattacharyya coefficient, respectively. Observe that the Chernoff upperbound is tighter (but of the same order) than the Bhattacharyya bound (for $\alpha^{*} \neq \frac{1}{2}$ ). Those upper bounds improve over the naive $\frac{1}{2}$ bound.

\begin{tabular}{ll|llll} 
dimension & $\lambda$ & $\widehat{P_{e}}$ & $\rho_{*}^{\mathrm{MVT}}$ & $\widehat{\alpha^{*}}$ & $\rho^{\mathrm{MVT}}$ \\
\hline$d=2$ & $\lambda=3$ & 0.3302 & 0.4471817 & 0.455 & 0.4475742 \\
$d=3$ & $\lambda=4$ & 0.2578 & 0.3951277 & 0.462 & 0.3956298 \\
$d=5$ & $\lambda=6$ & 0.16215 & 0.2943599 & 0.487 & 0.2944589 \\
$d=10$ & $\lambda=11$ & 0.06045 & 0.1400655 & 0.548 & 0.141438 \\
$d=15$ & $\lambda=16$ & 0.02845 & 0.07442729 & 0.592 & 0.07841622 \\
$d=20$ & $\lambda=21$ & 0.0167 & 0.04396945 & 0.625 & 0.04945252
\end{tabular}

we may assume wlog. that one parameter matrix is the identity while the other is diagonal.) We report the experimental results in Table 1 .

\section{Conclusion}

In this paper, we first reported a formula relating the Bayes error $B_{e}$ (including the probability of error $P_{e}$ ) to the total variation metric TV defined on scaled distributions (see Theorem 1). Second, we elucidated the Chernoff upper bound mechanism based on generalized weighted means: Chernoff [4] used the fact that $\min (a, b) \leq a^{\alpha} b^{1-\alpha}$ for $a, b>0$ and $\alpha \in[0,1]$ to derive an upper bound that turned out to be well-suited to the structure of exponential families [11. We interpreted the right-hand side of this inequality as a weighted geometric mean, and considered extending the upper bound construction using generalized weighted mean $M(a, b ; \alpha)$. A mean $M(a, b ; \alpha)$ is indeed guaranteed to fall within its extrema by definition, thus yielding the bounds: $\min (a, b) \leq M(a, b ; \alpha) \leq \max (a, b)$. We considered the family of quasi-arithmetic means [1, 12, 14] and showed how to derive new upper bounds by coupling the structure of the generalized mean with the structure of the probability distribution family at hand. We illustrated our method by considering three examples: The univariate Cauchy distributions, and the multivariate Pearson type VII and $t$-distributions (that includes the multivariate normal distributions in the limit case). For those families, we designed new affinity coefficients upper bounding $B_{e}$. The best value $\alpha^{*}$ of $\alpha$ yielding the tightest coefficient can be found by optimization on the statistical manifold [15. We carried out numerical experiments that show that those novel upper bounds are helpful because not too distant to Bayes error (although not very tight), specially because they can be calculated in constant time using closed-form formula. Otherwise, for more precise approximations, the Bayes error can be estimated using computationally-intensive stochastic integrations.

Last but not least, this paper revealed novel interactions between the Bayes error and statistical divergences: We show how to design $P_{e}\left(p_{1}, p_{2}\right) \leq \rho_{\alpha}^{f}\left(p_{1}, p_{2}\right)$ upper bounds where $\rho_{\alpha}^{f}$ is an affinity coefficient derived from a weighted quasi-arithmetic mean $M_{f}$ (skewed for Chernoff type and symmetric for a Bhattacharrya type). Since we can transform any affinity coefficient $\rho_{\alpha}^{f}$ into a corresponding divergence by defining $D_{\alpha}^{f}=-\log \rho_{\alpha}^{f}$, we deduce that those novel statistical divergences $D_{\alpha}^{f}$ can be used to upper bound the probability of error: $P_{e}\left(p_{1}, p_{2}\right) \leq e^{-D_{\alpha}^{f}\left(p_{1}, p_{2}\right)}$.

\section{Acknowledgments}

The author is grateful for the valuable comments of the Reviewers that led to this revised work. 


\section{A Bayes error for class-conditional Gaussians}

We summarize the formula for the Bayes error $B_{e}$ and the probability of error $P_{e}$ when dealing with univariate and multivariate normal class-conditional distributions:

$$
\begin{aligned}
B_{e} & =\frac{a_{1}+a_{2}}{2}-\operatorname{TV}\left(a_{1} p_{1}, a_{2} p_{2}\right), \\
P_{e} & =\frac{1}{2}-\operatorname{TV}\left(w_{1} p_{1}, w_{2} p_{2}\right),
\end{aligned}
$$

where $a_{1}=w_{1}\left(c_{11}+c_{21}\right)$ and $a_{2}=w_{2}\left(c_{12}+c_{22}\right)$ are the positive weights derived from the cost design matrix and the a priori class weights.

First, consider the family of univariate normal distributions $\mathcal{F}=\left\{N(\mu, \sigma) \mid \mu \in \mathbb{R}, \sigma \in \mathbb{R}_{+}\right\}$. Consider two distinct normal densities $p_{1}$ and $p_{2}$. The two weighted densities $a_{1} p_{1}(x)=a_{2} p_{2}(x)$ intersect at exactly two positions when $\sigma_{1} \neq \sigma_{2}$ or in exactly one position, otherwise:

$$
\frac{a_{1}}{\sigma_{1} \sqrt{2 \pi}} e^{-\frac{1}{2}\left(\frac{x-\mu_{1}}{\sigma_{1}}\right)^{2}}=\frac{a_{2}}{\sigma_{2} \sqrt{2 \pi}} e^{-\frac{1}{2}\left(\frac{x-\mu_{2}}{\sigma_{2}}\right)^{2}} .
$$

Finding the roots amounts to solve the quadratic equation:

$$
\left(\frac{x-\mu_{1}}{\sigma_{1}}\right)^{2}-\left(\frac{x-\mu_{2}}{\sigma_{2}}\right)^{2}-2 \log \frac{a_{1} \sigma_{2}}{\sigma_{1} a_{2}}=0 .
$$

When $\sigma_{1}=\sigma_{2}=\sigma\left(\right.$ with $\left.\mu_{1} \neq \mu_{2}\right)$, we have one root:

$$
x_{1}=\frac{\mu_{1}^{2}-\mu_{2}^{2}-2 \log \frac{a_{1} \sigma_{2}}{\sigma_{1} a_{2}}}{2\left(\mu_{1}-\mu_{2}\right)} .
$$

We find:

$$
\operatorname{TV}\left(a_{1} p_{1}, a_{2} p_{2}\right)=\frac{1}{2}\left|a_{2} \Phi\left(x_{1} ; \mu_{2}, \sigma_{2}\right)-a_{1} \Phi\left(x_{1} ; \mu_{1}, \sigma_{1}\right)\right|,
$$

where $\Phi(x ; \mu, \sigma)=\frac{1}{2}\left(1+\operatorname{erf}\left(\frac{x-\mu}{\sigma \sqrt{2}}\right)\right)$ is the cumulative distribution, and $\operatorname{erf}(x)=\frac{1}{\sqrt{\pi}} \int_{-x}^{x} e^{-t^{2}} \mathrm{~d} t$ denotes the error function. That is,

$$
\begin{aligned}
B_{e} & =\frac{a_{1}+a_{2}}{2}-\frac{1}{2}\left|a_{2} \operatorname{erf}\left(\frac{x_{1}-\mu_{2}}{\sigma \sqrt{2}}\right)-a_{1} \operatorname{erf}\left(\frac{x_{1}-\mu_{1}}{\sigma \sqrt{2}}\right)\right|, \\
x_{1} & =\frac{\mu_{1}^{2}-\mu_{2}^{2}-2 \log \frac{a_{1} \sigma_{2}}{\sigma_{1} a_{2}}}{2\left(\mu_{1}-\mu_{2}\right)} .
\end{aligned}
$$

When $\sigma_{1} \neq \sigma_{2}$, the quadratic equation expands as $a x^{2}+b x+c=0$, and we have two distinct roots $x_{1}$ and $x_{2}$ :

$$
\begin{aligned}
a & =\frac{1}{\sigma_{1}^{2}}-\frac{1}{\sigma_{2}^{2}}, \\
b & =2\left(\frac{\mu_{2}}{\sigma_{2}}-\frac{\mu_{1}}{\sigma_{1}}\right) \\
c & =\left(\frac{\mu_{1}}{\sigma_{1}}\right)^{2}-\left(\frac{\mu_{2}}{\sigma_{2}}\right)^{2}-2 \log \frac{a_{1} \sigma_{2}}{a_{2} \sigma_{1}} \\
x_{1} & =\frac{-b-\sqrt{\Delta}}{2 a}, \quad x_{2}=\frac{-b+\sqrt{\Delta}}{2 a},
\end{aligned}
$$


with $\Delta=b^{2}-4 a c \geq 0$ and the total variation writes as follows:

$$
\begin{aligned}
& \operatorname{TV}\left(a_{1} p_{1}, a_{2} p_{2}\right)= \\
& \quad \frac{1}{2}\left(\left|\operatorname{erf}\left(\frac{x_{1}-\mu_{1}}{\sigma_{1} \sqrt{2}}\right)-\operatorname{erf}\left(\frac{x_{1}-\mu_{2}}{\sigma_{2} \sqrt{2}}\right)\right|+\left|\operatorname{erf}\left(\frac{x_{2}-\mu_{1}}{\sigma_{1} \sqrt{2}}\right)-\operatorname{erf}\left(\frac{x_{2}-\mu_{2}}{\sigma_{2} \sqrt{2}}\right)\right|\right)
\end{aligned}
$$

Those formula generalize the probability of error reported in [3, p. 1375 to the most general case.

Second, consider the family of multivariate normals distributions $\left\{N(\mu, \Sigma) \mid \mu \in \mathbb{R}^{d}, \Sigma \succ 0\right\}$. For densities $p_{1}$ and $p_{2}$ having the same covariance matrix $\Sigma$, the probability of error is reported in [17, even for degenerate covariance matrices $\Sigma$ by taking the pseudo-inverse matrix $\Sigma^{+}$:

$$
P_{e}=\frac{1}{2}-\frac{1}{2} \operatorname{erf}\left(\frac{1}{2 \sqrt{2}}\left\|\left(\Sigma^{+}\right)^{\frac{1}{2}}\left(\mu_{2}-\mu_{1}\right)\right\|\right) .
$$

When covariance matrices are distinct $\left(\Sigma_{1} \neq \Sigma_{2}\right)$ but linear classifiers are considered, we also get a closed-form formula [18 for the probability of error. Otherwise, for the general case of quadratic classifiers of multivariate normals with distinct covariance matrices, no analytical formula is known. The best way to compute the probability of error is then by performing $1 D$ integration of the conditional density of the discriminant function $\mathbf{7}$.

Note that since two matrices can always be simultaneously diagonalized [7, it is enough to consider the case of two Gaussians with the first covariance being set to the identity matrix $I$ and the second covariance matrix set to a diagonal matrix $\Lambda$.

\section{References}

[1] János D. Aczél. On mean values. Bulletin of the American Mathematical Society, 54(4):392-400, 1948.

[2] Jorge M. Arevalillo and Hilario Navarro. A study of the effect of kurtosis on discriminant analysis under elliptical populations. Journal of Multivariate Analysis, 107:53-63, May 2012.

[3] Chin-Chun Chang and Tzung-Ying Lin. Linear feature extraction by integrating pairwise and global discriminatory information via sequential forward floating selection and kernel QR factorization with column pivoting. Pattern Recognition, 41(4):1373-1383, April 2008.

[4] Herman Chernoff. A measure of asymptotic efficiency for tests of a hypothesis based on the sum of observations. Annals of Mathematical Statistics, 23:493-507, 1952.

[5] Thomas Cover and Joy A. Thomas. Elements of information theory. Wiley-Interscience, 1991.

[6] F. Escolano, P. Suau and B. Bonev. Information Theory in Computer Vision and Pattern Recognition. Springer, 2009.

[7] Keinosuke Fukunaga. Introduction to statistical pattern recognition. Academic Press Professional, Inc., 1990. 2nd ed. (1st ed. 1972).

[8] Alan Genz, Frank Bretz, Tetsuhisa Miwa, Xuefei Mi, Friedrich Leisch, Fabian Scheipl and Torsten Hothorn. mvtnorm: Multivariate Normal and $t$ Distributions. http://CRAN.R-project.org/package=mvtnorm, 2013.

[9] Alan Genz and Frank Bretz. Computation of Multivariate Normal and t Probabilities. Lecture Notes in Statistics, 2009.

[10] Martin E. Hellman and Josef Raviv. Probability of error, equivocation and the Chernoff bound. IEEE Transactions on Information Theory, 16:368-372, 1970. 
[11] Thomas Kailath. The Divergence and Bhattacharyya distance measures in signal selection. IEEE Transactions on Communications, 15(1):52-60, 1967.

[12] Andrey Nikolaevich Kolmogorov. Sur la notion de la moyenne. Accad. Naz. Lincei Mem. Cl. Sci. Fis. Mat. Natur. Sez., 12:388-391, 1930.

[13] Christophe Ley and Anouk Neven. The normalizing constant in multivariate $t$-distributions: Dimension one versus higher dimensions. Technical report, 2012. 1211.1174.

[14] Mitio Nagumo. Über eine Klasse der Mittelwerte. Japanese Journal of Mathematics, 7:71-79, 1930. see Collected papers, Springer 1993.

[15] Frank Nielsen. An information-geometric characterization of Chernoff information. IEEE Signal Processing Letters, 20(3):269-272, March 2013.

[16] Frank Nielsen and Sylvain Boltz. The Burbea-Rao and Bhattacharyya centroids. IEEE Transactions on Information Theory, 57(8):5455-5466, August 2011.

[17] Mohammad Hossein Rohban, Prakash Ishwar, Birant Orten, William C. Karl, and Venkatesh Saligrama. An impossibility result for high dimensional supervised learning. 2013. arXiv/1301.6915.

[18] Luis Rueda. A one-dimensional analysis for the probability of error of linear classifiers for normally distributed classes. Pattern Recognition, 38(8):1197-1207, 2005.

[19] Jianyong Sun, Ata Kabán, and Jonathan M. Garibaldi. Robust mixture clustering using Pearson type VII distribution. Pattern Recognition Letters, 31(16):2447-2454, December 2010.

[20] Jianxin Wu and James M. Rehg. Beyond the Euclidean distance: Creating effective visual codebooks using the histogram intersection kernel. In International Conference on Computer Vision (ICCV), pages 630-637, 2009.

[21] Alan L. Yuille and James M. Coughlan. Fundamental Limits of Bayesian Inference: Order Parameters and Phase Transitions for Road Tracking. IEEE Transactions on Pattern Analysis and Machine Intelligence 22(2):160-173, February 2000.

\section{A $R$ code}

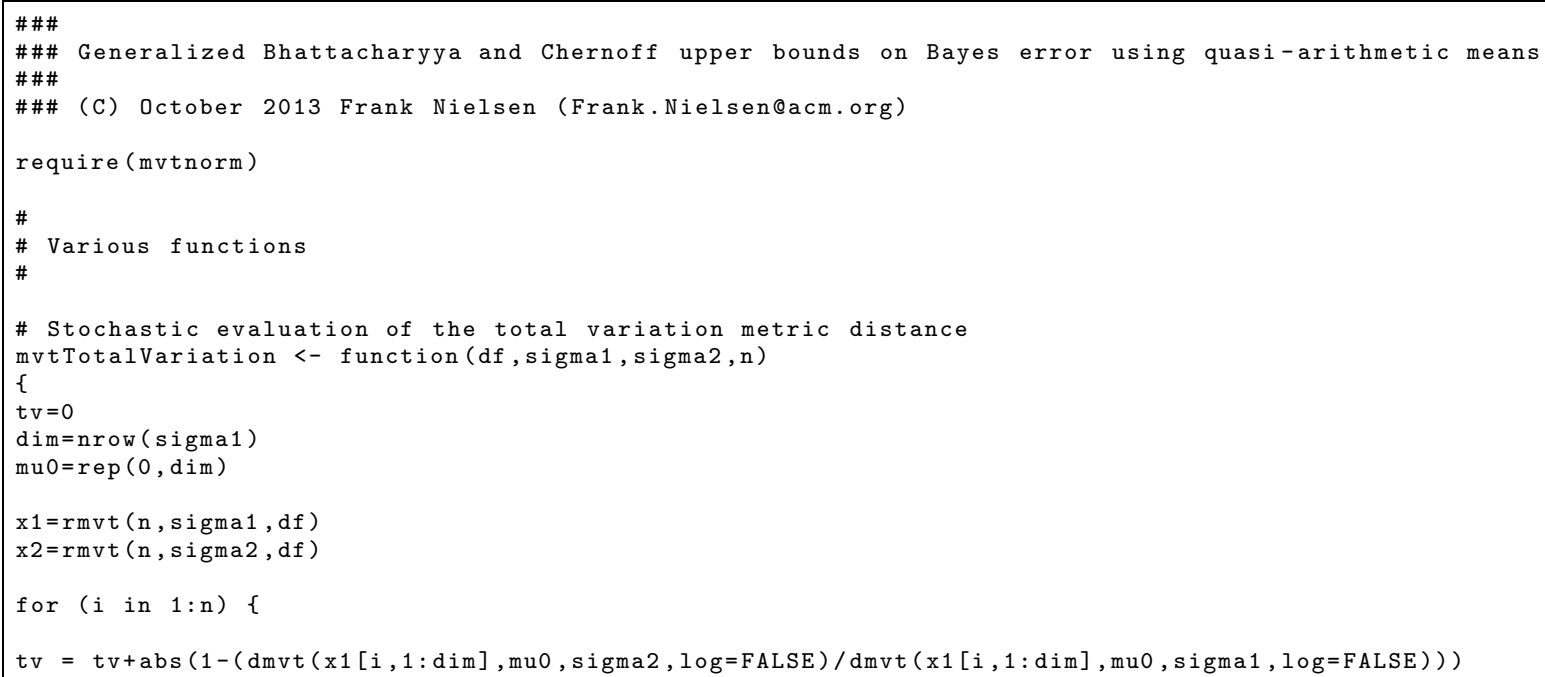




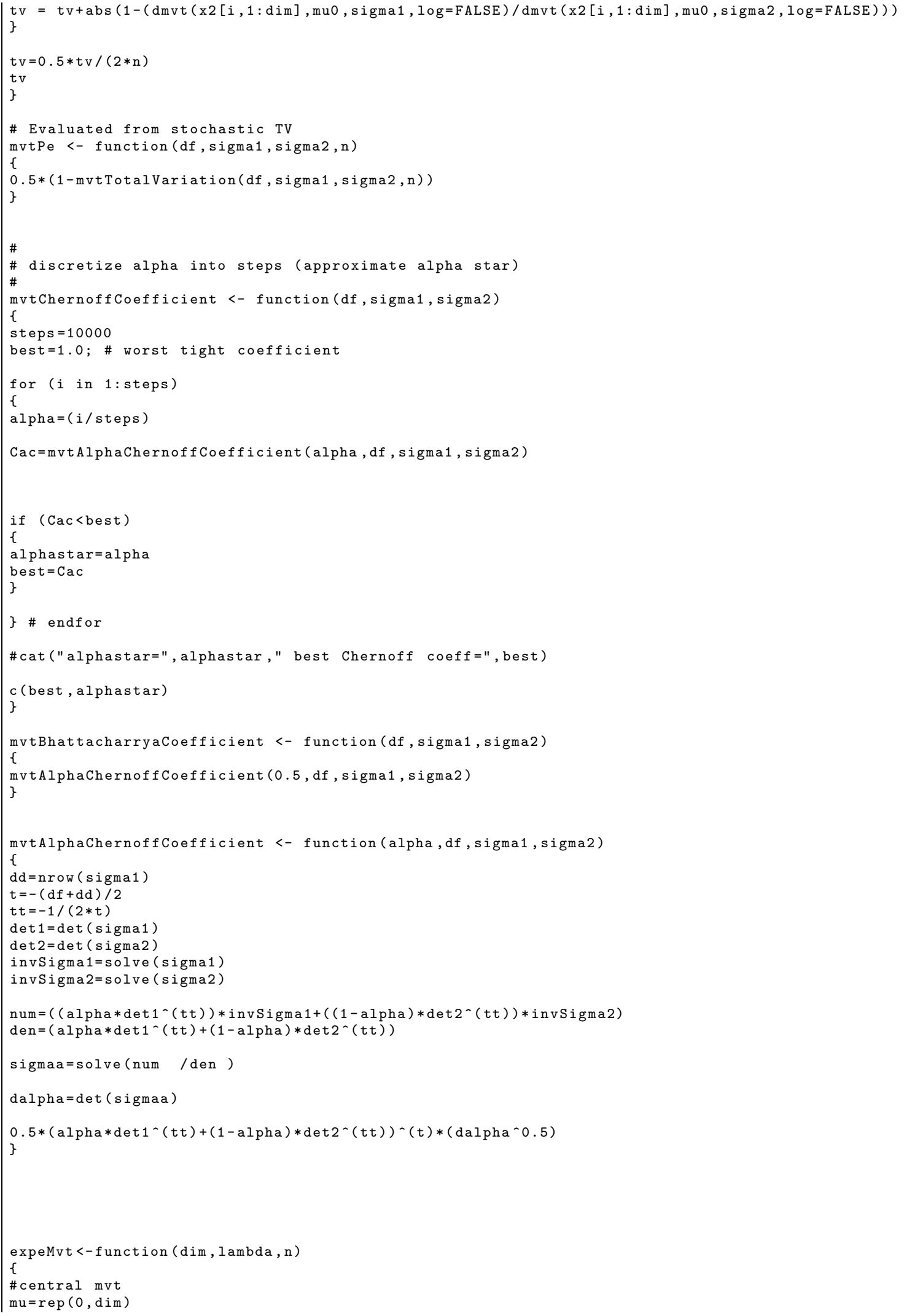




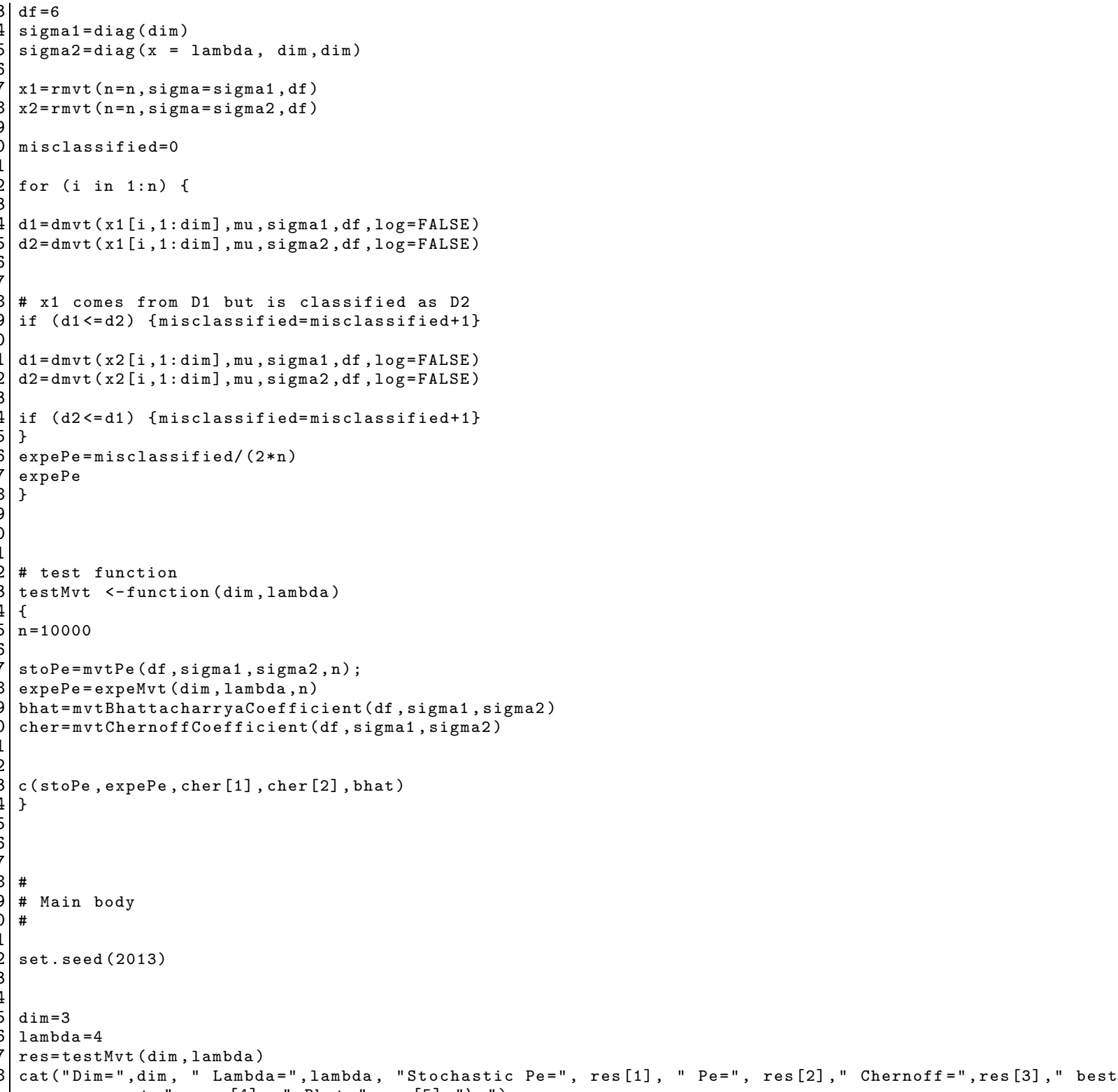

TestMVR.R 